\title{
Variations in oxidative stress markers in elite basketball players at the beginning and end of a season
}

\author{
YPATIOS SPANIDIS $^{1}$, NIKOLAOS GOUTZOURELAS ${ }^{1}$, DIMITRIOS STAGOS ${ }^{1}$, \\ ANASTASIOS MPESIOS ${ }^{1}$, ALEXANDROS PRIFTIS ${ }^{1}$, DAVID BAR-OR ${ }^{2-5}$, \\ DEMETRIOS A. SPANDIDOS ${ }^{6}$, ARISTIDES M. TSATSAKIS ${ }^{7}$, \\ GEORGE LEON $^{8}$ and DEMETRIOS KOURETAS ${ }^{1}$
}

\begin{abstract}
${ }^{1}$ Department of Biochemistry and Biotechnology, University of Thessaly, Larissa 41221, Greece; ${ }^{2}$ Department of Trauma Research, St. Anthony Hospital, Lakewood, CO 80228; ${ }^{3}$ Department of Trauma Research, Swedish Medical Center, Englewood, CO 80113; ${ }^{4}$ Department of Trauma Research, Medical Center of Plano, Plano, TX 75075; ${ }^{5}$ Luoxis Diagnostics, Inc., Englewood, CO 80112, USA; ${ }^{6}$ Laboratory of Clinical Virology, University of Crete, Medical School, Heraklion 71409; ${ }^{7}$ Department of Forensic Sciences and Toxicology, Medical School, University of Crete, Heraklion 71003; ${ }^{8}$ Standard Centre of Bioassays, 'Hartografoi Hygeias', Athens 15124, Greece
\end{abstract}

Received October 5, 2015; Accepted November 4, 2015

DOI: $10.3892 / \mathrm{etm} .2015 .2843$

\begin{abstract}
The aim of the present study was to examine the changes occuring in the redox status in male basketball players at the beginning and end of a highly competitive season. For this purpose, the redox status of 14 professional athletes of a European basketball club was examined at 2 different time points, at the beginning (phase 1) and at the end of the season (phase 2). The redox status was assessed in blood using conventional oxidative stress markers, such as thiobarbituric acid reactive substances (TBARS), protein carbonyls (CARB) and the total antioxidant capacity (TAC) in plasma, as well as glutathione (GSH) levels and catalase (CAT) activity in erythrocytes. Moreover, a new static oxidation-reduction potential marker (sORP) was assessed in plasma. Our results revealed that sORP was significantly increased by $9.6 \%$ and GSH levels were significantly decreased by $35.0 \%$ at phase 2 compared to phase 1 , indicating the induction of oxidative stress due to excessive exercise. Moreover, TAC was significantly increased by $12.9 \%$ at phase 2 compared to phase 1 , indicating the activation of adaptive responses for counteracting oxidative
\end{abstract}

Correspondence to: Professor Demetrios Kouretas, Department of Biochemistry and Biotechnology, University of Thessaly, Ploutonos 26 and Aiolou Street, Larissa 41221, Greece

E-mail: dkouret@uth.gr

Abbreviations: CAT, catalase; EDTA, ethylenediaminetetraacetic acid; GSH, glutathione; $\mathrm{H}_{2} \mathrm{O}_{2}$, hydrogen peroxide; ROS, reactive oxygen species; sORP, static oxidation-reduction potential; TAC, total antioxidant capacity; TBA, thiobarbituric acid; TBARS, thiobarbituric acid reactive substances; TCA, trichloroacetic acid

Key words: basketball, oxidative stress, oxidation-reduction potential, glutathione stress. The CARB and TBARS levels were not significantly altered between the 2 phases, although there was a significant correlation $(r=0.798)$ between the sORP and CARB levels. Furthermore, the variations in these markers between athletes were examined. We found that 3 markers exhibited a similar response between athletes, that is, sORP was increased in all 14 athletes, TAC was increased in 13 and the GSH levels were decreased in 14. However, the other 3 markers (i.e., TBARS, CARB and CAT) exhibited marked variations between the athletes, suggesting that the optimal approach with which to counteract (e.g., antioxidant supplementation) the observed increase in oxidative stress is the individualized examination of the redox status of athletes using a series of markers. This would allow the identification of athletes affected by severe oxidative stress and inflammation, and would thus indicate when necessary intervention measures are required to improve their health and performance.

\section{Introduction}

Basketball is considered one of the most popular team sports worldwide and has gained the fascination of countless spectators due to its dynamic characteristics. The overall duration of a typical basketball match is $40-48 \mathrm{~min}$, in which an athlete carries out a combination of multidirectional movements, such as running, jumping and dribbling at variable velocities. Even though both aerobic and anaerobic systems are activated in order to execute these movements, previous studies have demonstrated that the anaerobic metabolism is the primary energy pathway activated in basketball players (1-4).

Elite basketball athletes undergo heavy training and competition throughout the season, continously playing numerous difficult and demanding matches. It has been well established that intense exercise increases the production of free radicals, which may lead to a pathophysiological condition known as oxidative stress $(5,6)$ that has been implicated in the oxidative 
damage to macromolecules (e.g., lipids, proteins and DNA) (7), immune dysfunction (8), muscle damage (9) and fatigue (10). It is well known that oxidative stress frequently occurs in muscle tissue exposed to reactive oxygen species (ROS) production (8). During intense exercise, there is a high rate of $\mathrm{O}_{2}$ consumption in skeletal muscle, which, due to the incomplete $\mathrm{O}_{2}$ reduction and electron leakage from the electron transfer chain, then leads to the accumulation of mitochondrial-derived ROS (8). These, in combination with the extra-mitochondrial produced ROS may cause oxidative stress (11). These effects in turn result in muscle fatigue, cell damage and apoptosis $(11,12)$. A previous study demonstrated that the intensity of a basketball game varies depending on the level of the competition and the playing position (13). In addition, other studies have indicated the induction of oxidative stress in elite team-sport athletes over the course of an athletic season, particularly in periods of intense competition $(14,15)$.

In the present study, an elite European basketball professional team was monitored at the beginning and end of an athletic season to examine the differences in the redox status in players between the beginning of the season and following a series of highly competitive matches at the end of the season. In order to monitor the redox status of the athletes, conventional oxidative stress markers, such as thiobarbituric acid reactive substances (TBARS), glutathione (GSH) levels, catalase (CAT) activity, protein carbonyl (CARB) levels and total antioxidant capacity (TAC) were measured. Moreover, a novel method based on the measurement of oxidation-reduction potential (ORP) was used for assessing oxidative stress. ORP is an integrated measure of the balance between total oxidants [e.g., oxidized thiols, superoxide radicals, hydroxyl radicals, hydrogen peroxide $\left(\mathrm{H}_{2} \mathrm{O}_{2}\right)$, nitric oxide, peroxynitrite and transition metal ions] and total reductants (e.g., free thiols, ascorbate, $\alpha$-tocopherol, $\beta$-carotene and uric acid), as well as other unknown markers (16). In previous studies, we demonstrated that the measurement of ORP using the RedoxSYS diagnostic system was an effective method for assessing oxidative stress induced by strenuous exercise, such as a mountain marathon race and eccentric exercise $(17,18)$.

\section{Subjects and methods}

Subjects. A total of 14 adult male basketball players (age, $26.8 \pm 1.2$ years; height, $1.99 \pm 0.02 \mathrm{~m}$; weight, $101.6 \pm 2.63 \mathrm{~kg}$ ) participated in the present study. All players were members of an elite European professional basketball club that participates in both European and national basketball leagues. All experimental procedures were carried out in accordance with the European Union Guidelines laid down in the 1964 Declaration of Helsinki and were approved by the Ethics Committee of the University of Thessaly (Larissa, Greece).

Training load. The measurements were carried out on 2 different selected time points of a basketball championship season (32 weeks), which presented differences in the tiredness and fatigue of the athletes. Thus, blood collections were made at 2 different phases, at the beginning of the regular season (phase 1; November) and at the end of the season (phase 2; May) following a series of high-level competitive matches. In phase 1 , the team had already played 9 matches over the course of 1 month, having 2 matches per week. In phase 2 , the team had played 59 matches, while over the last 10 days prior to blood sampling, the players had participated in 4 consecutive and very intense games.

Blood collection. The blood samples were collected by the Health Centre 'Hartografoi Ygeias' (Athens, Greece), stored in ethylenediaminetetraacetic acid (EDTA) or heparin tubes and centrifuged at $1,370 \mathrm{x} \mathrm{g}$ for $10 \mathrm{~min}$ at $4^{\circ} \mathrm{C}$ to divide the erythrocytes from the plasma. The packed erythrocytes were lysed with 1:1 (v/v) distilled water, inverted vigorously and centrifuged at $4,020 \times \mathrm{g}$ for $15 \mathrm{~min}$ at $4^{\circ} \mathrm{C}$. The plasma and erythrocyte lysates were then stored at $-80^{\circ} \mathrm{C}$ until use in biochemical analysis.

Blood assays. The static ORP (sORP) marker was determined using the RedoxSYS diagnostic system (Luoxis Diagnostics, Inc., Englewood, CO, USA) as previously described $(17,18)$. This value is indicative of the integrated balance of oxidants and reductants in a specimen and is presented in $\mathrm{mV}$. Using this innovative method, $20 \mu \mathrm{l}$ of plasma were applied to disposable sensors designed by Luoxis Diagnostics, Inc., which were inserted into the RedoxSYS diagnostic system and the sORP value was reported within $4 \mathrm{~min}$.

For the determination of the levels of TBARS, an assay was used based on the study by Keles et al (19). TBARS is a commonly and frequently used method to determine the lipid peroxidation (20). In accordance with this method, $100 \mu \mathrm{l}$ of plasma were mixed with $500 \mu \mathrm{l}$ of $35 \%$ trichloroacetic acid (Merck KGaA, Darmstadt, Germany) and $500 \mu \mathrm{l}$ of Tris-HCl (Sigma-Aldrich, St. Louis, MO, USA; 200 mmol/1, pH 7.4) followed by incubation for $10 \mathrm{~min}$ at room temperature. A total of $1 \mathrm{ml}$ of $2 \mathrm{M}$ sodium sulfate and $55 \mathrm{mmol} / 1 \mathrm{TBA}$ solution were added and the samples were then incubated at $95^{\circ} \mathrm{C}$ for $45 \mathrm{~min}$. The samples were cooled on ice for $5 \mathrm{~min}$ and were vortexed following the addition of $1 \mathrm{ml}$ of $70 \%$ TCA. The samples were centrifuged at $15,000 \mathrm{x} \mathrm{g}$ for $3 \mathrm{~min}$ and the absorbance of the supernatant was read at $530 \mathrm{~nm}$ using a spectrophotometer (Hitachi U-1900; serial no. 2023-029; Hitachi, Tokyo, Japan). A baseline absorbance was taken into account by running a blank along with all samples during the measurement. The calculation of the TBARS concentration was based on the molar extinction co-efficient of malondialdehyde.

The GSH concentration was measured as previously described in the study by Reddy et al (21). A total of $20 \mu \mathrm{l}$ of erythrocyte lysate treated with 5\% TCA was mixed with $660 \mu \mathrm{l}$ of $67 \mathrm{mmol} / \mathrm{l}$ sodium potassium phosphate ( $\mathrm{pH} \mathrm{8.0)} \mathrm{and} 330 \mu \mathrm{l}$ of $1 \mathrm{mmol} / 1$ 5,5'-dithiobis-(2-nitrobenzoic acid) (DTNB). The samples were then incubated in the dark at room temperature for $45 \mathrm{~min}$ and the absorbance was read at $412 \mathrm{~nm}$ using a spectrophotometer (Hitachi U-1900; serial no. 2023-029; Hitachi). The GSH concentration was calculated on the basis of calibration curves made using commercial standards.

The concentration of CARB, an index of protein oxidation, was determined based on the method described in the study by Patsoukis et al (22). In this assay, $50 \mu 1$ of $20 \%$ TCA were added to $50 \mu \mathrm{l}$ of plasma and this mixture was then incubated in an ice bath for $15 \mathrm{~min}$ and centrifuged at 15,000 $\mathrm{x} \mathrm{g}$ for $5 \mathrm{~min}$ at $4^{\circ} \mathrm{C}$. The supernatant was discarded and $500 \mu \mathrm{l}$ of $10 \mathrm{mmol} / \mathrm{l}$ 2,4-dinitrophenylhydrazine (DNPH; in $2.5 \mathrm{~N} \mathrm{HCl}$ ) 
for the sample, or $500 \mu \mathrm{l}$ of $2.5 \mathrm{~N} \mathrm{HCl}$ for the blank, were added to the pellet. The samples were incubated in the dark at room temperature for $1 \mathrm{~h}$ with intermittent vortexing every $15 \mathrm{~min}$ and were centrifuged at $15,000 \mathrm{xg}$ for $5 \mathrm{~min}$ at $4^{\circ} \mathrm{C}$. The supernatant was discarded and $1 \mathrm{ml}$ of $10 \%$ TCA was added, vortexed and centrifuged at $15,000 \mathrm{xg}$ for $5 \mathrm{~min}$ at $4^{\circ} \mathrm{C}$. The supernatant was discarded and $1 \mathrm{ml}$ of ethanol-ethyl acetate $(1: 1 \mathrm{v} / \mathrm{v})$ was added, vortexed and centrifuged at $15,000 \mathrm{x} \mathrm{g}$ for $5 \mathrm{~min}$ at $4^{\circ} \mathrm{C}$. This washing step was repeated twice. The supernatant was discarded and $1 \mathrm{ml}$ of $5 \mathrm{M}$ urea ( $\mathrm{pH} \mathrm{2.3)} \mathrm{was} \mathrm{added,} \mathrm{vortexed}$ and incubated at $37^{\circ} \mathrm{C}$ for $15 \mathrm{~min}$. The samples were centrifuged at $15,000 \mathrm{x}$ g for $3 \mathrm{~min}$ at $4^{\circ} \mathrm{C}$ and the absorbance was read at $375 \mathrm{~nm}$ using a spectrophotometer (Hitachi U-1900; serial no. 2023-029; Hitachi). The calculation of the CARB concentration was based on the molar extinction co-efficient of DNPH. Total plasma protein was assayed using Bradford reagent (Sigma, Hamburg, Germany).

The determination of TAC was based on the method described in the study by Janaszewska and Bartosz (23). Briefly, $20 \mu \mathrm{l}$ of plasma were added respectively to $480 \mu \mathrm{l}$ of $10 \mathrm{mmol} / \mathrm{l}$ sodium potassium phosphate (pH 7.4) and $500 \mu 1$ of $0.1 \mathrm{mmol} / 1$ 1,1-diphenyl-1-picrylhydrazyl (DPPH) and the samples were incubated in the dark for $60 \mathrm{~min}$ at room temperature. The samples were then centrifuged for $3 \mathrm{~min}$ at $20,000 \mathrm{x}$ g and the absorbance was read at $520 \mathrm{~nm}$ using a spectrophotometer (Hitachi U-1900; serial no. 2023-029; Hitachi).

The measurement of CAT activity was carried out as previously described by Aebi (24). In particular, $4 \mu 1$ of erythrocyte lysate (diluted 1:10) were added to $2,991 \mu$ l of $67 \mathrm{mmol} / \mathrm{l}$ sodium potassium phosphate $(\mathrm{pH} \mathrm{7.4)}$ and the samples were incubated at $37^{\circ} \mathrm{C}$ for $10 \mathrm{~min}$. A total of $5 \mu \mathrm{l}$ of $30 \% \mathrm{H}_{2} \mathrm{O}_{2}$ was added to the samples and the change in absorbance was immediately read at $240 \mathrm{~nm}$ [using a spectrophotometer (Hitachi U-1900; serial no. 2023-029; Hitachi)] for $130 \mathrm{sec}$. The calculation of CAT activity was based on the molar extinction co-efficient of $\mathrm{H}_{2} \mathrm{O}_{2}$. Each assay was performed twice in triplicate.

Statistical analysis. For statistical analysis, data were analyzed by one-way ANOVA followed by Dunnett's test for multiple pairwise comparisons. The correlation between different oxidative stress markers was examined by Spearman's correlation analysis. A value of $\mathrm{P}<0.05$ was considered to indicate a statistically significant difference. For all statistical analyses, SPSS version 13.0 software (SPSS, Inc., Chicago, IL, USA) was used. Data are presented as the means \pm standard error of the mean.

\section{Results}

The results revealed that the sORP value, which is indicative of the current redox status, increased significantly $(\mathrm{P}<0.05)$ by $9.6 \%$ at phase 2 compared to phase 1 (Fig. 1). As regards the other oxidative stress markers, the CARB and TBARS levels which are indicative of protein oxidation and lipid peroxidation, respectively were not significantly affected (Fig. 2A and B). However, TAC was significantly increased $(\mathrm{P}<0.05)$ by $12.9 \%$ (Fig. 2C) at phase 2 compared to phase 1 . In addition, the GSH levels in erythrocytes were significantly decreased $(\mathrm{P}<0.05)$ by $35 \%$ at phase 2 compared to phase 1 (Fig. 3A), whereas CAT activity was not affected (Fig. 3B).

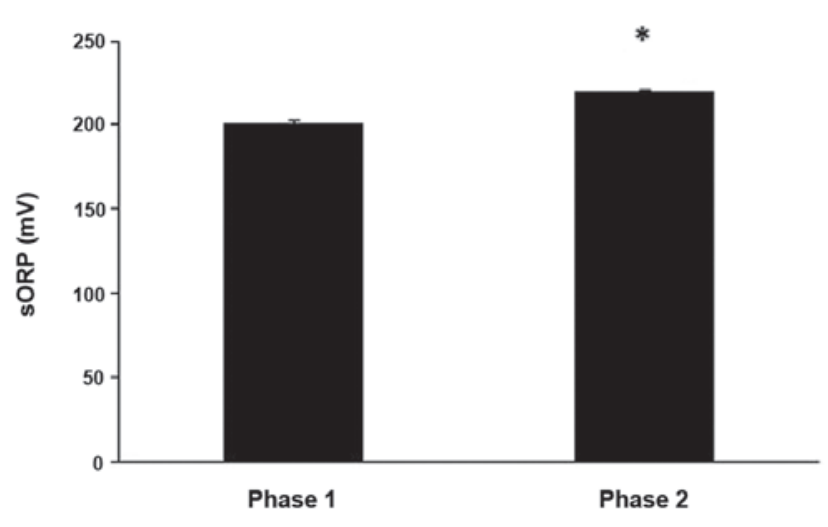

Figure 1. Values (means \pm standard error of the mean) of the oxidative stress marker, static oxidation-reduction potential (sORP), in the plasma of basketball players at the beginning (phase 1) and end of season (phase 2). ${ }^{*} \mathrm{P}<0.05$, significantly different compared with phase 1 .
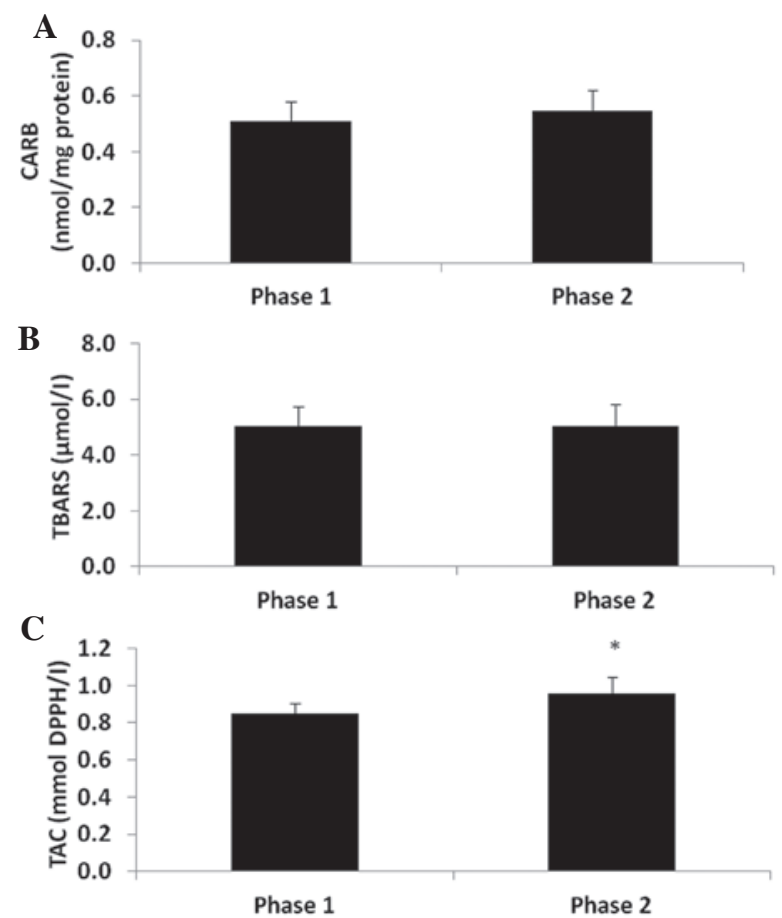

Figure 2. Values (means \pm standard error of the mean) of oxidative stress markers in plasma of basketball players at the beginning (phase 1) and end of season (phase 2). (A) Protein carbonyl (CARB) levels, (B) thiobarbituric acid reactive substances (TBARS) levels, (C) total antioxidant capacity (TAC). ${ }^{*} \mathrm{P}<0.05$, significantly different compared with phase 1 .

Since there is growing evidence provided by ours and previous studies that there is a marked heterogeneity in responses between different individuals to exercise-induced oxidative stress $(17,25-27)$, in this study, we examined the changes occurring in oxidative stress markers in each individual player between phase 1 and 2 of the athletic season (Fig. 4). We found that in all players, the sORP values were higher at phase 2 compared to phase 1 (Fig. 4A). Similar to the sORP values, the TAC values were altered in a similar manner in almost all players at phase 2 compared to phase 1, apart from one player (Fig. 4B). However, the changes in the levels of 2 other markers measured in plasma, CARB and TBARS, exhibited marked variations between the players (Fig. 4C and D). 
A
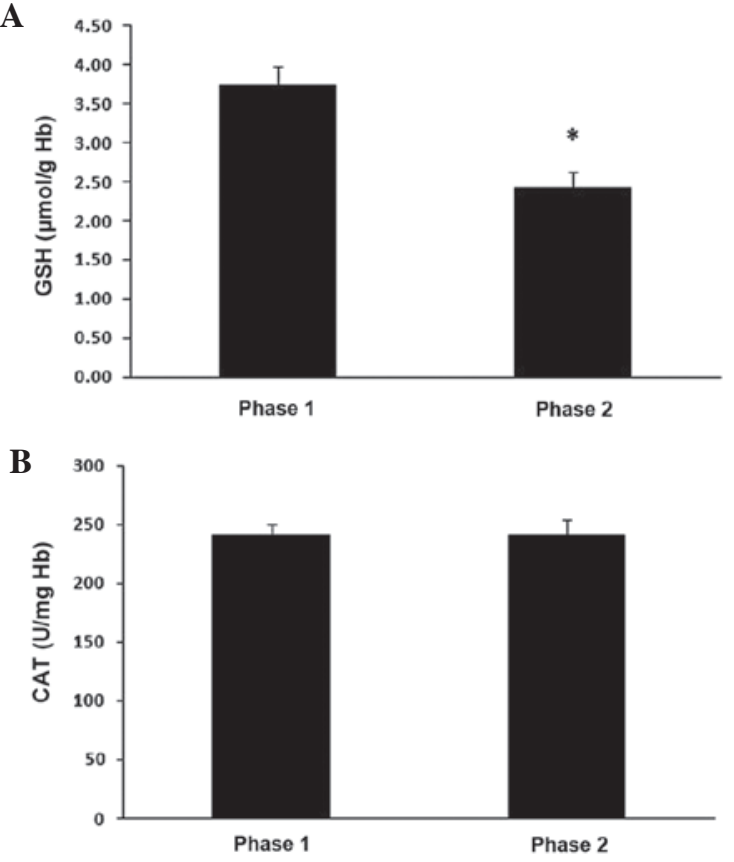

Figure 3. Values (means \pm standard error of the mean) of oxidative stress markers in erythrocytes of basketball players at the beginning (phase 1) and end of season (phase 2). (A) Glutathione (GSH) levels, (B) catalase (CAT) activity. ${ }^{*} \mathrm{P}<0.05$, significantly different compared with phase 1 .
Specifically, half of the players exhibited an increase in CARB and TBARS levels at phase 2 compared to phase 1 , whereas the remaining players exhibited a decrease (Fig. 4C and D). As regards the markers measured in erythrocytes, the GSH concentration was decreased in all players at phase 2 compared to phase 1 (Fig. 4E). On the contrary, the changes in CAT activity presented marked differences between players, since in 6 players, there was an increase in enzymatic activity at phase 2 compared to phase 1 , while in 8 players there was a decrease (Fig. 4F).

Spearman's correlation analysis between percentage changes of the oxidative stress markers revealed that there was only a statistically significant $(\mathrm{P}<0.001)$ high correlation $(r=0.798)$ between the sORP and CARB markers (Table I and Fig. 5).

\section{Discussion}

To the best of our knowledge, there are only a few studies available to date on the assessment of the redox status of basketball players, particularly over the course of a season $(28,29)$. In this study, the redox status of 14 elite basketball athletes was examined at 2 different time points, at the beginning of a season (phase 1) and at the end of a season (phase 2). At phase 1, the players had only played 9 matches. However, at phase 2 , the
A

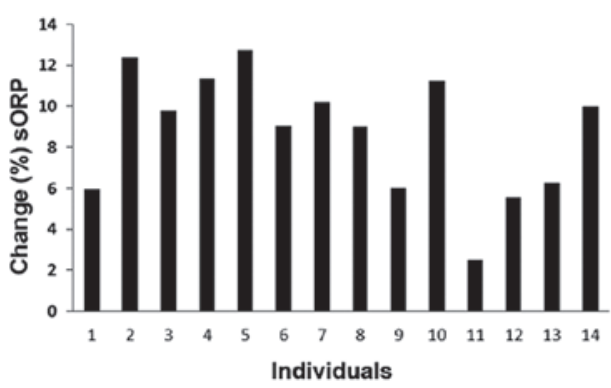

C
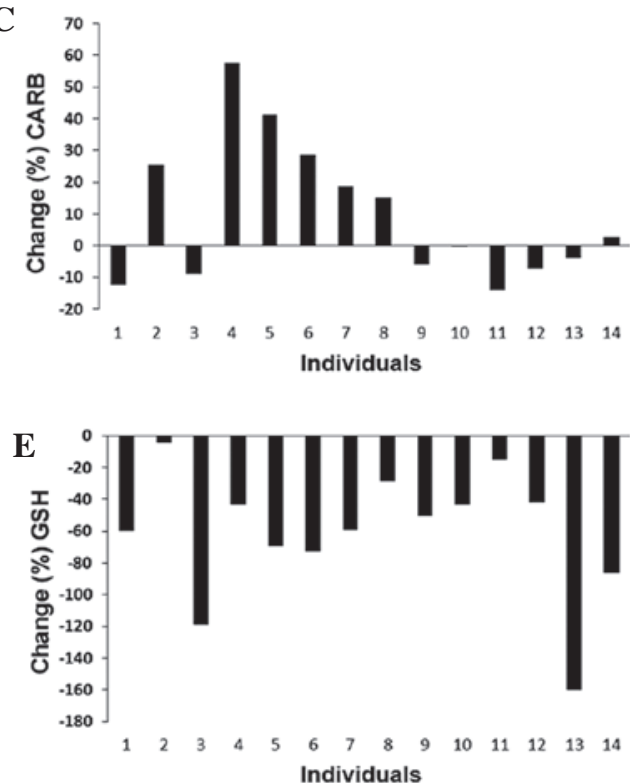

B

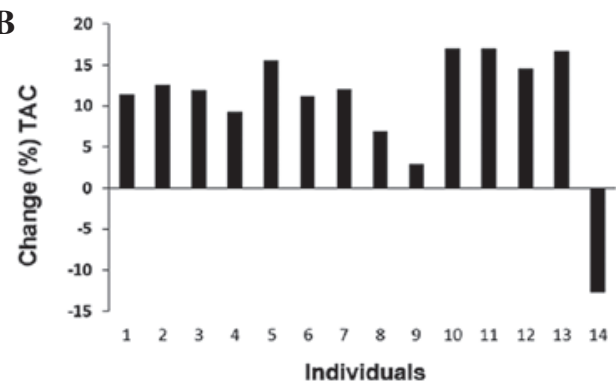

D
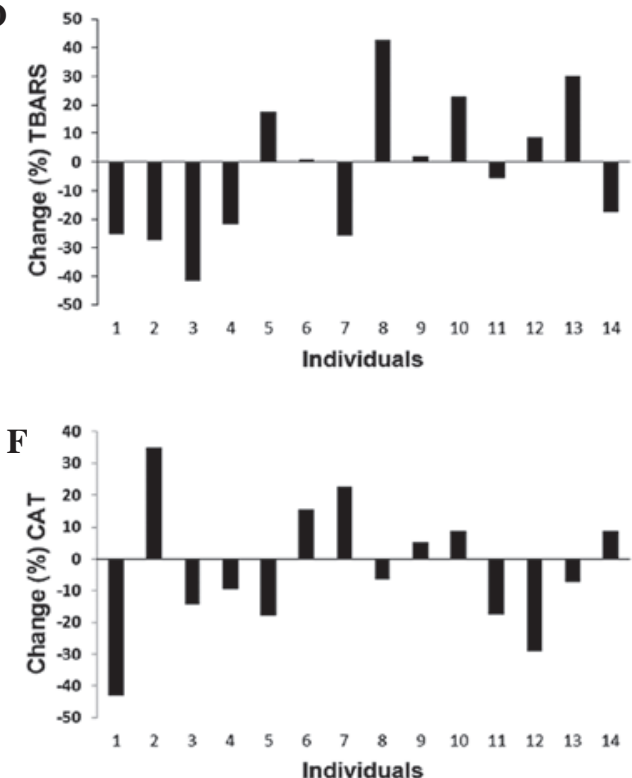

Figure 4. Percentage changes in oxidative stress biomarkers of each individual at the end of season (phase 2) compared to the beginning of season (phase 1). (A) Static oxidation-reduction potential (sORP) (in plasma), (B) total antioxidant capacity (TAC) (in plasma), (C) protein carbonyl (CARB) (in plasma), (D) thiobarbituric acid reactive substances (TBARS) (in plasma), (E) glutathione (GSH) (in erythrocytes) and (F) catalase (CAT) activity (in erythrocytes). Each bar represents the percentage difference in the level of each marker between phase 2 and phase 1. 
Table I. Correlation co-efficient (r) of percentage change (i.e., change between phase 1 and phase 2) between the sORP, TAC, CARB, TBARS and CAT markers.

\begin{tabular}{|c|c|c|c|c|c|c|}
\hline Markers & sORP & TAC & TBARS & CARB & GSH & CAT \\
\hline sORP & & 0.011 & -0.191 & $0.798^{\mathrm{a}}$ & -0.064 & 0.455 \\
\hline TAC & & & 0.209 & -0.213 & 0.182 & -0.165 \\
\hline TBARS & & & & 0.055 & 0.051 & -0.125 \\
\hline CARB & & & & & -0.007 & 0.477 \\
\hline GSH & & & & & & 0.095 \\
\hline
\end{tabular}

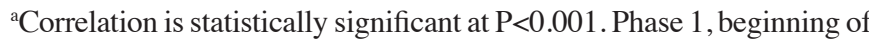
season; phase 2, end of season; sORP, tatic oxidation-reduction potential marker; TAC, total antioxidant capacity; CARB, protein carbonyls; TBARS, thiobarbituric acid reactive substances; CAT, catalase; GSH, glutathione.

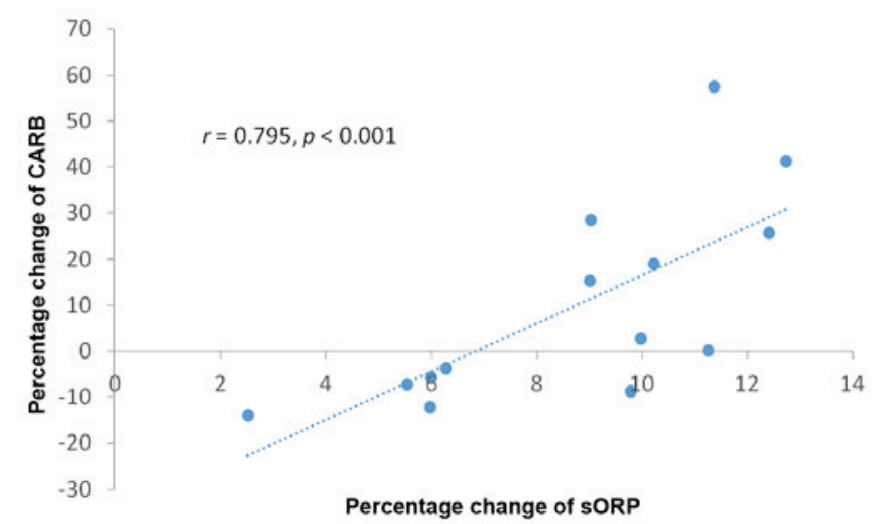

Figure 5. Correlation of percentage change [i.e., change between the beginning (phase 1) and end of season (phase 2)] between static oxidation-reduction potential (sORP) and protein carbonyl (CARB).

players had played 59 competitive matches, a number which is considered extremely high for a European team.

Thus, the excessive amounts of exercise due to the high number of matches at phase 2 resulted in more severe oxidative stress compared to phase 1. Specifically, the GSH levels in erythrocytes were significantly lower in the athletes at phase 2 than in phase 1 . It has previously been reported that acute aerobic exercise results in a decrease in GSH levels due in part to the inactivation of free radicals for regenerating ascorbic acid and $\alpha$-tocopherol (14). Of note, a similar study conducted by Zembron-Lacny et al (28), in professional players of the Polish Basketball Extraleague, demonstrated that the GSH levels were also decreased at the end of the playoff period. This increase in GSH levels was accompanied by an increase in the levels of anti-inflammatory interleukin-6 (IL-6) and pro-inflammatory tumor necrosis factor- $\alpha$ (TNF- $\alpha$ ) cytokines (28). In general, it has been proposed that the exercise-induced increase in ROS activates adaptive responses through signaling pathways regulated by the thiol status (i.e., reduced and oxidized GSH levels) (28-31). Changes in the thiol status induce the expression of the transcription factors, nuclear factor- $\mathrm{kB}$ and activator protein-1 (AP-1), which in turn increase the levels of the cytokines, IL-6 and TNF- $\alpha$ (30-32). Finally, IL- 6 and TNF- $\alpha$ play crucial role in muscle regeneration and in the development of tolerance following ROS-induced muscle damage (33). In this study, in contrast to the GSH levels, the activity of CAT in erythrocytes did not differ between the 2 phases. However, a recent study reported that CAT activity increased after a basketball game (34). In general, the mechanisms through which exercise affects CAT activity are not clear, although studies have indicatd that CAT activity is not increased after exercise (35-38).

Moreover, in this study, the oxidative stress which occurred at phase 2 was not so severe in order to cause lipid peroxidation and protein oxidation, as evidenced by the unaltered TBARS and CARB levels. Similarly, Zembron-Lacny et al (28) did not observe any increase in the TBARS and CARB levels in basketball players at the end of the playoff round. The hypothesis accounting for this absence of lipid peroxidation and protein oxidation is that exercise-induced ROS generation increases antioxidant defense mechanisms through changes in the thiol status as mentioned above (28).

The above mentioned results of the present study demonstrating a decrease in GSH levels were also confirmed by the increase in the values of the sORP marker. This marker as measured by the RedoxSYS diagnostic system, was used in order to evaluate oxidative stress in basketball players. sORP is the standard potential between a working electrode and a reference electrode with no driving current (or extremely small current) which is proportional to the balance of reductants and oxidants in plasma (17). Low sORP values mean that the biological sample is in the normal range of redox status, while high sORP values mean that the biological sample is in a higher state of oxidative stress. We have previously reported that the sORP value was increased in athletes following a mountain marathon race, consequently indicating the induction of oxidative stress (18). Similarly, in this study, the increase in the values of the sORP marker at phase 2 compared to phase 1 suggested an increase in oxidative stress. Moreover, the percentage change in the values of the sORP marker between phase 1 and 2 had a high correlation with the changes in the CARB levels, although as mentioned above, there was no statistically significant difference in the CARB levels at phase 2 compared to phase 1 . This finding suggests that the higher the oxidative stress, the higher the probability for protein oxidation in athletes and also supports the use of sORP as a novel marker of oxidative stress.

The other marker which was used to assess the total redox status was TAC. TAC was higher in the athletes at phase 2 compared to phase 1, indicating an enhancement of the antioxidant mechanisms at the end of the season. This result seems intriguing, particularly when compared to the induction of oxidative stress suggested by other biomarkers. Other studies, including ours have also reported an increase in TAC postexercise (18,39-41). This apparent contradiction is explained if we bear in mind that TAC actually assesses the total amount of molecules acting as antioxidants. Indeed, previous studies have reported that exercise enhances the antioxidant mechanisms $(42,43)$. However, as we have previously noted (18), the increase in TAC following exercise may indicate that this method is inappropriate for assessing the in vivo redox status. 
The weakness of TAC as a method is that it is based on the reduction of a free radical (e.g., DPPH) by the antioxidant molecules in plasma. Thus, this method evaluates only the reductants in plasma. However, the redox status is determined not only by the amount of reductants, but also by the amount of oxidants in plasma. Thus, sORP may be a better marker than TAC for assessing the total oxidative stress in vivo, since its assessment is based on both the amount of reductants and oxidants in plasma.

In a previous study, we also examined the use of ORP markers for assessing eccentric exercise-induced oxidative stress and found a marked variation in the changes of oxidative stress markers between different individuals (17). In general, the issue of examining not only average group responses to exercise-induced oxidative stress, but also individual responses is of great interest, since previous research has also recently reported great inter-individual variability regarding the changes in oxidative stress markers following exercise (26). The present findings also exhibited great inter-individual variations in the changes of oxidative stress markers in athletes between phase 1 and 2 . Thus, the 6 tested oxidative stress markers could be divided into 2 categories based on their changes at phase 2 compared to phase 1.

The first category includes the 3 oxidative stress markers (i.e., sORP, TAC and GSH) in which all (or almost all) the athletes exhibited the same directional variations at phase 2 compared to phase 1 . Thus, the sORP value was increased in all athletes, GSH levels were decreased in all athletes and TAC was increased in all but one athlete at phase 2 compared to phase 1 . The increase in the sORP value in all athletes indicated that oxidative stress occurred in all athletes due to excessive exercise at phase 2 . However, there was great inder-individual variability in the percentage change of sORP at phase 2 compared to phase 1 (the least percentage change was $2.5 \%$ and the highest was $12.7 \%$ ). The increase in TAC in all but one athlete at phase 2 compared to phase 1 conformed with the increase in the SORP value, since it suggested an increase in antioxidant molecules (i.e., reductants) due to adaptive response to oxidative stress induced at phase 2. Although the concurrence of the increase in TAC with oxidative stress is intriguing, it may explained by the fact that oxidative stress occurs when there is an imbalance between oxidants and reductants in favour of the former (44). Thus, a stimulus may cause an increase in the amount of both oxidants and reductants, but if the former are higher than the latter, then oxidative stress will occur. For this reason, we believe that the sORP marker is more effective than TAC for assessing the redox status, since it is based on the evaluation of the difference between oxidants and reductants. Similar to the sORP value, TAC also exhibited great inder-individual variability in the percentage change (the least percentage increase was $2.9 \%$ and the highest was $17.0 \%$ ). The decrease in TAC in 1 athlete at phase 2 compared to phase 1 may be explained by his inability to effectively activate adaptive responses to oxidative stress. The decrease in the GSH levels in all athletes at phase 2 compared to phase 1 may indicate the crucial role that this antioxidant molecule plays in exercise-induced oxidative stress. As mentioned above, there is evidence that oxidative stress induced by exercise activates adaptive responses through signaling pathways regulated by the thiol status $(30,31)$.
The second category includes the 3 oxidative stress markers (i.e., TBARS, CARB and TAC) that exhibited either a decrease or increase among different athletes at phase 2 compared to phase 1 . The marked variation in the levels of these markers may be explained by the high complexity of the regulation of redox homeostasis in humans. Previous studies have reported that different factors, such as genetic, physiological, biochemical and dietary factors affect the final effects of oxidative stress $(27,41)$.

In conclusion, the findings of the present study demonstrated that oxidative stress is induced in basketball players at the beginning and end of a season due to excessive exercise. The most important effect was the increase in the sORP value and TAC and the decrease in GSH levels in the athletes. The decrease in GSH levels occurs particularly when exercise induces muscle damage (45). Basketball athletes are subjected to muscle injury due to the high numbers of jumps and sprints during a basketball game (46). Muscle-damaging exercise induces inflammation which at low levels, in turn, helps the muscle regeneration (41). However, exercise-induced inflammation at high levels can cause health problems and affect the performance of athletes (47). Adaptive responses are also induced due to this oxidative stress as shown by an increase in TAC. Moreover, the sORP marker has been shown to be effective for monitoring the redox status in basketball athletes, as previously demonstrated for other types of exercise (18). On the other hand, there was a great variation in the oxidative damage to biological macromolecules (e.g., protein oxidation, lipid peroxidation and CAT) between the different athletes. From the above-mentioned findings, it can be inferred that the optimal intervention approach with which (e.g., antioxidant supplementation) to reduce the detrimental effects of exercise-induced oxidative stress on human health is the individualized examination of the redox status in athletes using different markers. This would allow the identification of athletes affected by severe oxidative stress and inflammation, and they would thus be administered the appropriate antioxidant supplementation. On the other hand, athletes who present low levels of exercise-induced oxidative stress and inflammation may not require antioxidant intervention in order for the antioxidant adaptive mechanisms to be activated and exert their beneficial effects.

Our research group has performed the individualized monitoring of exercise-induced oxidative stress in athletes for several years. We believe that currently a crucial point regarding exercise-induced oxidative stress is to determine the optimal threshold of the oxidative stress level above which the appropriate antioxidant supplementation should be used in order to help each athlete improve his health status and performance.

\section{References}

1. Ciuti C, Marcello C, Macis A, Onnis E, Solinas R, Lai C and Concu A: Improved aerobic power by detraining in basketball players mainly trained for strength. Sports Med Train Rehabil 6: 325-335, 1996.

2. Balčiūnas M, Stonkus S, Abrantes C and Sampaio J: Long term effects of different training modalities on power, speed, skill and anaerobic capacity in young male basketball players. J Sports Sci Med 5: 163-170, 2006.

3. Crisafulli A, Melis F, Tocco F, Laconi P, Lai C and Concu A: External mechanical work versus oxidative energy consumption ratio during a basketball field test. J Sports Med Phys Fitness 42: 409-417, 2002. 
4. McInnes SE, Carlson JS, Jones CJ and McKenna MJ: The physiological load imposed on basketball players during competition. J Sports Sci 13: 387-397, 1995

5. Veskoukis AS, Nikolaidis MG, Kyparos A, Kokkinos D, Nepka C, Barbanis S and Kouretas D: Effects of xanthine oxidase inhibition on oxidative stress and swimming performance in rats. Appl Physiol Nutr Metab 33: 1140-1154, 2008.

6. Nikolaidis MG, Jamurtas AZ, Paschalis V, Kostaropoulos IA Kladi-Skandali A, Balamitsi V, Koutedakis Y and Kouretas D: Exercise-induced oxidative stress in G6PD-deficient individuals. Med Sci Sports Exerc 38: 1443-1450, 2006.

7. Mylonas C and Kouretas D: Lipid peroxidation and tissue damage. In Vivo 13: 295-309, 1999.

8. Schneider BSP and Tiidus PM: Neutrophil infiltration in exercise-injured skeletal muscle: how do we resolve the controversy? Sports Med 37: 837-856, 2007.

9. Nikolaidis MG, Kyparos A, Hadziioannou M, Panou N, Samaras L, Jamurtas AZ and Kouretas D: Acute exercise markedly increases blood oxidative stress in boys and girls. Appl Physiol Nutr Metab 32: 197-205, 2007.

10. Meeus M, Nijs J, Hermans L, Goubert D and Calders P: The role of mitochondrial dysfunctions due to oxidative and nitrosative stress in the chronic pain or chronic fatigue syndromes and fibromyalgia patients: peripheral and central mechanisms as therapeutic targets? Expert Opin Ther Targets 17: 1081-1089, 2013.

11. Phaneuf S and Leeuwenburgh C: Apoptosis and exercise. Med Sci Sports Exerc 33: 393-396, 2001.

12. McClung JM, Deruisseau KC, Whidden MA, Van Remmen H, Richardson A, Song W, Vrabas IS and Powers SK: Overexpression of antioxidant enzymes in diaphragm muscle does not alter contraction-induced fatigue or recovery. Exp Physiol 95: 222-231, 2010.

13. Rodríguez-Alonso M, Fernández-García B, Pérez-Landaluce J and Terrados $\mathrm{N}$ : Blood lactate and heart rate during national and international women's basketball. J Sports Med Phys Fitness 43: 432-436, 2003

14. Finaud J, Lac G and Filaire E: Oxidative stress: relationship with exercise and training. Sports Med 36: 327-358, 2006.

15. Schippinger G, Fankhauser F, Abuja PM, Winklhofer-Roob BM, Nadlinger K, Halwachs-Baumann G and Wonisch W: Competitive and seasonal oxidative stress in elite alpine ski racers. Scand J Med Sci Sports 19: 206-212, 2009.

16. Spanidis Y, Goutzourelas N, Stagos D, Kolyva AS, Gogos CA, Bar-Or D and Kouretas D: Assessment of oxidative stress in septic and obese patients using markers of oxidation-reduction potential. In Vivo 29: 595-600, 2015.

17. Stagos D, Goutzourelas N, Ntontou AM, Kafantaris I, Deli CK, Poulios A, Jamurtas AZ, Bar-Or D and Kouretas D: Assessment of eccentric exercise-induced oxidative stress using oxidation-reduction potential markers. Oxid Med Cell Longev 2015: 204615, 2015.

18. Stagos D, Goutzourelas N, Bar-Or D, Ntontou AM, Bella E, Becker AT, Statiri A, Kafantaris I and Kouretas D: Application of a new oxidation-reduction potential assessment method in strenuous exercise-induced oxidative stress. Redox Rep 20: 154-162, 2015.

19. Keles MS, Taysi S, Sen N, Aksoy H and Akçay F: Effect of corticosteroid therapy on serum and CSF malondialdehyde and antioxidant proteins in multiple sclerosis. Can J Neurol Sci 28: 141-143, 2001.

20. Lykkesfeldt J: Malondialdehyde as biomarker of oxidative damage to lipids caused by smoking. Clin Chim Acta 380: 50-58, 2007.

21. Reddy YN, Murthy SV, Krishna DR and Prabhakar MC: Role of free radicals and antioxidants in tuberculosis patients. Indian J Tuberc 51: 213-218, 2004

22. Patsoukis N, Zervoudakis G, Panagopoulos NT, Georgiou CD, Angelatou F and Matsokis NA: Thiol redox state (TRS) and oxidative stress in the mouse hippocampus after pentylenetetrazol-induced epileptic seizure. Neurosci Lett 357: 83-86, 2004.

23. Janaszewska A and Bartosz G: Assay of total antioxidant capacity: comparison of four methods as applied to human blood plasma. Scand J Clin Lab Invest 62: 231-236, 2002.

24. Aebi H: Catalase in vitro. Methods Enzymol 105: 121-126, 1984.

25. Margaritelis NV, Veskoukis AS, Paschalis V, Vrabas IS, Dipla K, Zafeiridis A, Kyparos A and Nikolaidis MG: Blood reflects tissue oxidative stress: a systematic review. Biomarkers 20: 97-108, 2015

26. Margaritelis NV, Kyparos A, Paschalis V, Theodorou AA, Panayiotou G, Zafeiridis A, Dipla K, Nikolaidis MG and Vrabas IS: Reductive stress after exercise: The issue of redox individuality. Redox Biol 2: 520-528, 2014.
27. Rankinen T and Bouchard C: Gene-physical activity interactions: Overview of human studies. Obesity (Silver Spring) 16 (Suppl 3): S47-S50, 2008.

28. Zembron-Lacny A, Slowinska-Lisowska M and Ziemba A: Integration of the thiol redox status with cytokine response to physical training in professional basketball players. Physiol Res 59: 239-245, 2010.

29. Melikoglu MA, Kaldirimci M, Katkat D, Sen I, Kaplan I and Senel K: The effect of regular long term training on antioxidant enzymatic activities. J Sports Med Phys Fitness 48: 388-390, 2008.

30. Zembron-Lacny A, Naczk M, Gajewski M, Ostapiuk-Karolczuk J, Dziewiecka H, Kasperska A and Szyszka K: Changes of muscle-derived cytokines in relation to thiol redox status and reactive oxygen and nitrogen species. Physiol Res 59: 945-951, 2010.

31. Ji LL, Gomez-Cabrera MC and Vina J: Exercise and hormesis: activation of cellular antioxidant signaling pathway. Ann N Y Acad Sci 1067: 425-435, 2006.

32. Kerksick $\mathrm{C}$ and Willoughby D: The antioxidant role of glutathione and $\mathrm{N}$-acetyl-cysteine supplements and exercise-induced oxidative stress. J Int Soc Sports Nutr 2: 38-44, 2005.

33. Steensberg A, van Hall G, Osada T, Sacchetti M, Saltin B and Klarlund Pedersen B: Production of interleukin-6 in contracting human skeletal muscles can account for the exercise-induced increase in plasma interleukin-6. J Physiol 529: 237-242, 2000.

34. Chatzinikolaou A, Draganidis D, Avloniti A, Karipidis A, Jamurtas AZ, Skevaki CL, Tsoukas D, Sovatzidis A, Theodorou A, Kambas A, et al: The microcycle of inflammation and performance changes after a basketball match. J Sports Sci 32: 870-882, 2014.

35. Wiggs MP: Can endurance exercise preconditioning prevention disuse muscle atrophy? Front Physiol 6: 63, 2015.

36. Oh-ishi S, Kizaki T, Nagasawa J, Izawa T, Komabayashi T, Nagata N, Suzuki K, Taniguchi N and Ohno H: Effects of endurance training on superoxide dismutase activity, content and mRNA expression in rat muscle. Clin Exp Pharmacol Physiol 24: 326-332, 1997.

37. Smuder AJ, Kavazis AN, Min K and Powers SK: Exercise protects against doxorubicin-induced oxidative stress and proteolysis in skeletal muscle. J Appl Physiol 1985 110: 935-942, 2011

38. Mangner N, Linke A, Oberbach A, Kullnick Y, Gielen S, Sandri M, Hoellriegel R, Matsumoto Y, Schuler G and Adams V: Exercise training prevents TNF- $\alpha$ induced loss of force in the diaphragm of mice. PLoS One 8: e52274, 2013.

39. Fatouros IG, Jamurtas AZ, Villiotou V, Pouliopoulou S, Fotinakis P, Taxildaris K and Deliconstantinos G: Oxidative stress responses in older men during endurance training and detraining. Med Sci Sports Exerc 36: 2065-2072, 2004.

40. Wiecek M, Maciejczyk M, Szymura J and Szygula Z: Changes in oxidative stress and acid-base balance in men and women following maximal-intensity physical exercise. Physiol Res 64: 93-102, 2015 .

41. Bloomer RJ and Fisher-Wellman KH: Blood oxidative stress biomarkers: influence of sex, exercise training status, and dietary intake. Gend Med 5: 218-228, 2008

42. Gul M, Demircan B, Taysi S, Oztasan N, Gumustekin K, Siktar E, Polat MF, Akar S, Akcay F and Dane S: Effects of endurance training and acute exhaustive exercise on antioxidant defense mechanisms in rat heart. Comp Biochem Physiol A Mol Integr Physiol 143: 239-245, 2006

43. Powers SK and Jackson MJ: Exercise-induced oxidative stress: Cellular mechanisms and impact on muscle force production. Physiol Rev 88: 1243-1276, 2008.

44. Rahal A, Kumar A, Singh V, Yadav B, Tiwari R, Chakraborty S and Dhama K: Oxidative stress, prooxidants, and antioxidants: the interplay. BioMed Res Int 2014: 761264, 2014.

45. Nikolaidis MG, Jamurtas AZ, Paschalis V, Fatouros IG, Koutedakis Y and Kouretas D: The effect of muscle-damaging exercise on blood and skeletal muscle oxidative stress: magnitude and time-course considerations. Sports Med 38: 579-606, 2008.

46. Pliauga V, Kamandulis S, Dargevičiūtė G, Jaszczanin J, Klizienė I, Stanislovaitienè $J$ and Stanislovaitis A: The effect of a simulated basketball game on players' sprint and jump performance, temperature and muscle damage. J Hum Kinet 46: 167-175, 2015.

47. Pyne DB: Exercise-induced muscle damage and inflammation a review. Aust J Sci Med Sport 26: 49-58, 1994. 